laitière, depuis des siècles peut-être. Ses scurs de la Frise Orientale et de la Frise Hollandaise quoiqu'elles doivent passer des hivers rudes, sont des excellentes laitières, qui ne le cèdent en rien à la Flamande, car on note également un maximum de 4 et 5 litres de lait au mois de mai, et il serait à examiner si notre laitière ne donnerait pas plus dans le sud de la France. J'oserais l'affirmer, s'il ne restait pas une autre influence qui pourrait nuire à la lactation, notamment la vie en troupeaux. J'ai déjà dit que notre brebis vit en stabulation et toute sa constitution s'est transformée en ce sens, que notre brebis n'est peut-être pas aussi chercheuse et aussi brouteuse qu'il serait à souhaiter dans le pays calcaire et rocheux, à une altitude de 800 mètres.

Il s'agit donc d'améliorer le Larzac, comme la Flamande, par la sélection, et d'étudier les moyens de nourrir économiquement les sujets, afin d'en tirer le plus grand profit.

\title{
QUELQUES MOTS SUR LE LAIT DE GHEVRE,
}

par M. le Professeur GH. PORCHER. de l'Ecole vétérinaire de Lyon.

Ce n'est pas un examen complet du lait de chèvre que nous allons présenter ici. Il pourra trouver sa place une autre fois ; mais nous pensons qu'il est bon de profiter de ce que nous dirons ici pour rassembler quelques documents intéressants.

En relisant plusieurs des travaux qui ont trait au lait de chèvre, nous y avons vu avec surprise étaler cette opinion qu'il élait possible de modifier à volonté le lait de chèvre par l'alimentation, afin de le rapprocher du lait de femme. C'est une opinion contre laquelle nous nous élevons énergiquement.

Le lait de chèvre a sa figure propre, qui n'est pas aussi modifiable qu'on veut bien le dire par l'alimentation. Nous ne répèterons pas ici ce que nous avons dit ailleurs du lait de vache: c'est qu'il est nécessaire de séparer nettement la matière grasse des éléments non gras qui sont contenus dans l'extrait dégraissé. A celle-là, la variabilité, à ceux-ci la quasi-fixité.

La matière grasse, dans le lait de chèvre, comine elle le fait dans 

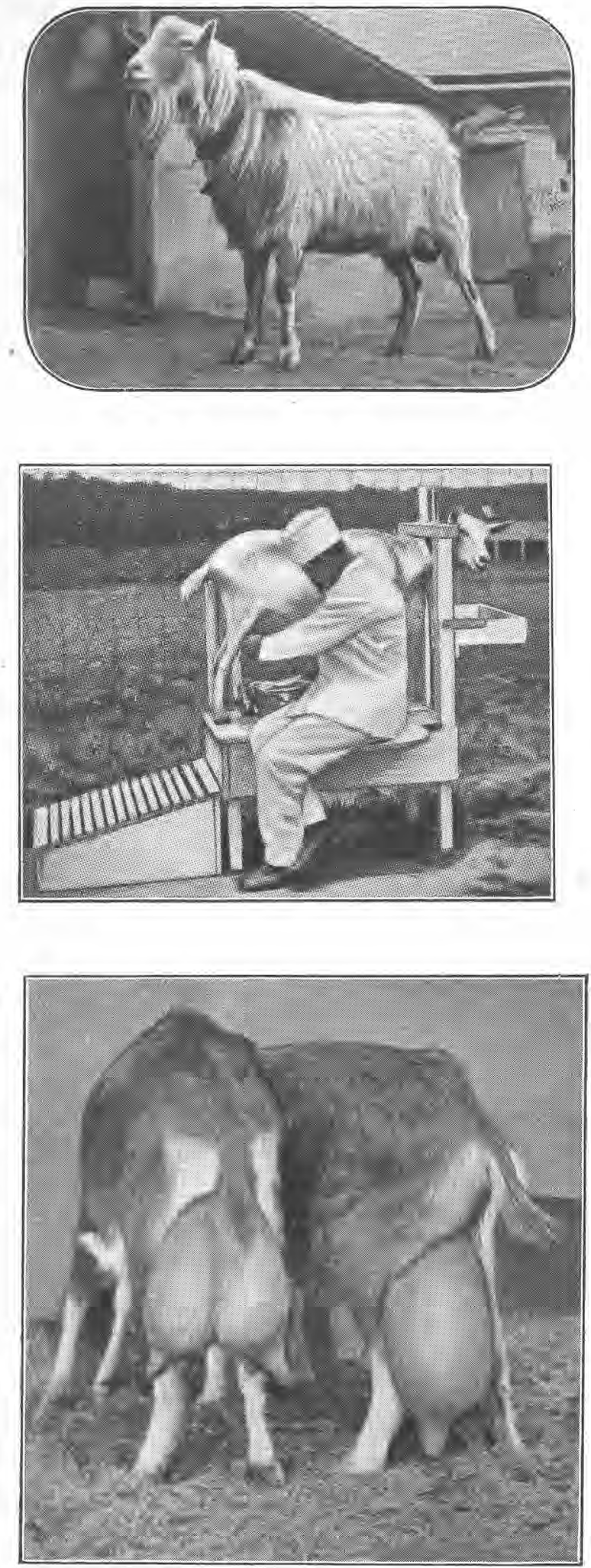

\section{Bouc producteur de lait}

(V. travail de J3. Macalik, dans ee numéro p. 334 .

\section{Petite estrade pour traite de la chèvre}

(Edw. L. Shaw., Farmer's, Bull. n" 920, Min. de l'dgr. des Etats-Unis).

Analyse daus ee numéro, p. 356.

Remarquable développement de mamelle chez deux chèvres qu ont servi à nourrir des enfant

(Voil Angora et Milk Goat Jomrall, Dec, 192 p. 35). 
le lait de vache, varie alors même que le régime alimentaire reste constant ; c'est pourquoi j'ai tenu, lors des discussions qui ont eu lieu à Ruremonde, à faire remarquer après la communication de $\mathrm{M}$. ZWagerman, que la matière grasse dans le lait de chèvre ne varie pas comme il voulait bien le dire, imperceptiblement, d'un jour à l'autre, mais que son taux, comme dans le lait de toutes les autres espèces d'ailleurs, éprouvait des variations pouvant être considérables du jour au lendemain, d'un animal à l'autre, etc... Les courbes et le tableau insérés dans ce travail le montrent remarquablement.

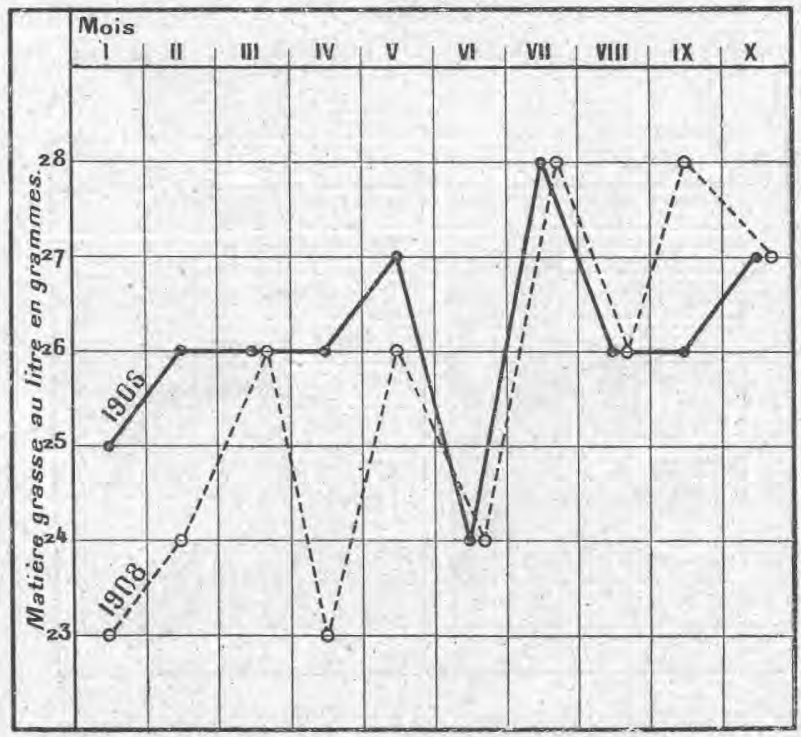

Moyenne butyreuse mensuelle du lait de mélange de 5 chèvres au cours de deux périodes de Iactalion (Instilut Zootechnique de l'Ecole vétérinaire de Dresde). - II s'agit de chèvres au lait peu riche en matière grasse. Noler que les deux courbes assez superposables sur leur droite ne le sont pas sur leur gauche.

Objectivement, le lait de chèvre est blanc parce que sa matière grasse n'est pas colorée; aussi le beurre que l'on en retire est-il très blanc et dans les pays à grosse production de lait de chèvre, il serait recommandable de colorer le produit obtenu, si une industrie beurrière venait à se développer.

Quant à sa composition chimique, nous pouvons dire que le lait de chèvre ressemble beaucoup au lait de vache, et les différences sur lesquelles, avec complaissance, insistent certains auteurs, ne sont pas si marquées qu'il veulent bien le dire. Le lait de chèvre, comme 
le lait de vache, est du type caséineux, ce qui veut dire que la caséïne prédomịne dans les matières protéiques, alors que le lait de femme et le lait des solipèdes (jument, ànesse) appartiennent au type albumineux, la proportion de l'albumine étant très élevée par rap. port à celle de la caséine.

Alors que dans le lait de vache on peut estimer à 35-36 grammes environ le taux moyen des protéiques totaux par litre, dans le lait de chèvre, ce dernier atteint 39 à 40 grammes. Si les quantités de caséine contenues dans ces deux laits sont à peu près les mêmes, il y a cependant - et c'est là la plus notable différence dans la composition chimique de ces deux laits, - une plus grande quantité d'albumine

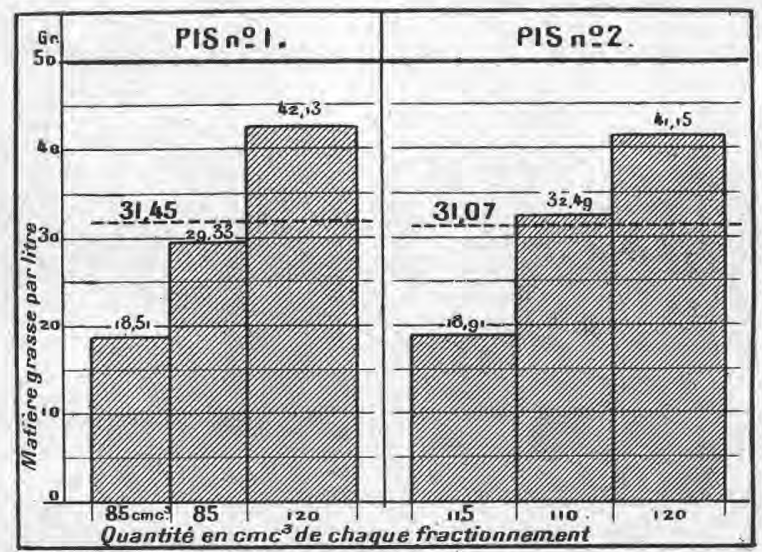

Taux, en grammes par litre, de la matière grasse dans le lait des trois fractionnements de la traite effectuée chez une chèvre, sur chaque quartier séparément (Thèse de Cailloux, Bordeaux, 1911-12.). - Coinme chez la femme, la vache, la richesse butyreuse va en augmentant du commencement à la fin de la traite.

dans le lait de chèvre que dans le lait de vache. C'est peut-être à ce fait que le lait de chèvre doit d'être un peu plus visqueux que le lait de vache. Nous ajouterons que, peut-être, faut-il lui attribuer en outre la propriété qu'a le lait de chèvre de donner avec la présure un caillot peut-être un peu plus diffluent, un peu plus mou que celui que fournit le lait de vache, car plus il y a d'albumine dans un lait, plus l'action de la présure est gênée.

Les globules du lait de chèvre sont notabilement plus petits que ceux du lait de vache ; le fait était déjà connu depuis longtemps, mais, dans un travail tout récent, E.-W. Schultz et L.-R. Ghand- 
LER (1) y reviennent à nouveau. Ces deux auteurs ont examiné 45 échantillons de lait de chèvre; le diamètre des globules gras était moindre que $4 \mu$ dans $91 \%$ des globules gras, moindre que $2 \mu$

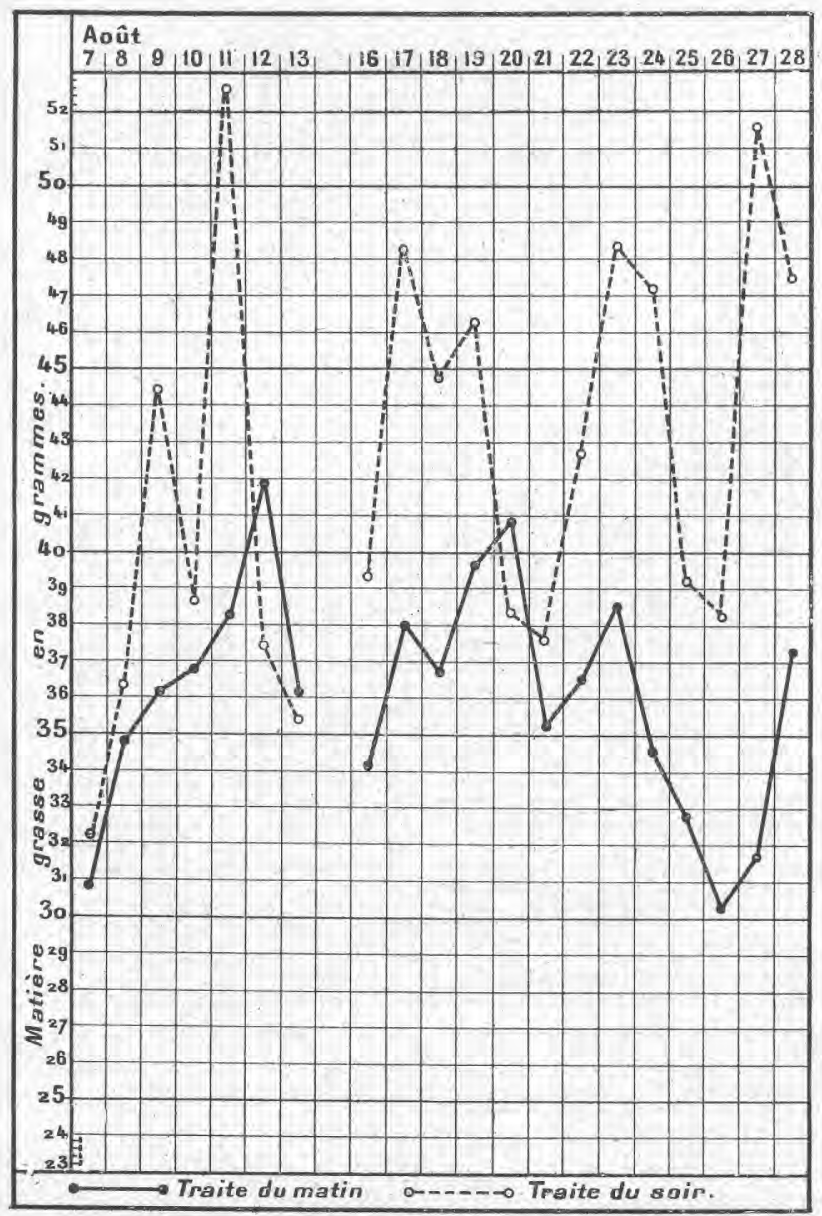

Taux, en grammes par litre, de la malière grasse du lait d'une chèvre au troisième mois de la lactation sur les deux trailes journalières (Thèse de CaILLoux). A noter que la traite du soir est, à trois exception près, sur 20 jours, plus riche en matière grasse que celle du malin.Remarquer également lez variations désordonnées du taux bulyreux du lait, qu'il s'agisse de la traite du matin ou de celle du soir, d'un jour à l'autre, si cn considère deux traites homologues et du. matin au soir, pour les deux traites du même jour.

dans $57 \%$, alors que, chez la vache, $90 \%$ des globules gras du lait dépassent $4 \mu$ de diamètre. La matière grasse est donc notablement

(1) Jonrnal of Biological Chemistry, T. 46, 1921, p. 133. 
plus dispersée chez la chèrre que chez la vache. D'autre part, le milieu étant plus visqueux chez la chèvre que chez la vache, on s'explique aussi que la montée de la crême se fasse plus difficilement, ainsi que l'expérience le montre.Néanmoins, en mettant en jeu l'écrémeuse centrifuge, on parvient à écrémer parfaitement le lait de chèvre.

La plus grande dispersion de la graisse dans le lait contribue sans doute aussi à sa plus facile digestion.

Si les anaIyses de laits de chèvre rencontrées dans certains livres paraissent parfois assez disparates, il faut penser en trouver l'explication dans ce fait que l'auteur ne dit pas toujours à quel moment de la lactation le lait a été examiné. La chèvre est un animal qu'on ne trait pas - et c'est un tort - avec la régularité qu'on apporte plus généralement dans la traite de la vache. Les phénomènes de rétention lactée que j'ai étudiés ces années dernières (1) interviennent vraisemblablement ici, et le chiffre faible de lactose signalé par certains auteurs y trouve peut-être là son explication.

Dans le travail que je rappelle, je parle longuement de l'effet de la multiplication des traites. Chez la chèvre très souvent, et notamment chez l'animal ambulant, le chevrier ne procède jamais à des traites régulières. Il trait l'animal à la demande du client qui se présente ; on a donc à faire à des traites répétées, mais nous ajouterons que la traite n'est jamais complète, et, par conséquent, l'effet de la multiplication des traites ne se manifeste pas dans toute sa pureté comme si chaque traite, si rapprochée qu'elle fût de la précédente, devait être complète.

Je rapporte ici le tableau p. 346 que j'ai publié dans mon travail sur la rétention lactée, son examen attentif nous montrera trois choses :

$1^{\circ}$ En multipliant les traites, on augmente légèrement la richesse du lait du fait que l'on supprime toute rétention possible;

$2^{\circ}$ La multiplication des traites aboutit à une augmentation notable de la production laitière, sans qu'il y ait pour cela amoindrissement de la richesse, c'est-à-dire diminution de la qualité chimique, tant au point de vue gras que non gras;

$3^{\circ}$ On pourra juger, chez l'animal dont il a été question dans ce tableau, de la très grande variabilité du taux de la matière grasse au litre.

(1) La Rétention La tée. Arch, de Mílecine des Enfunts, octobre et novombre 1.120 , 


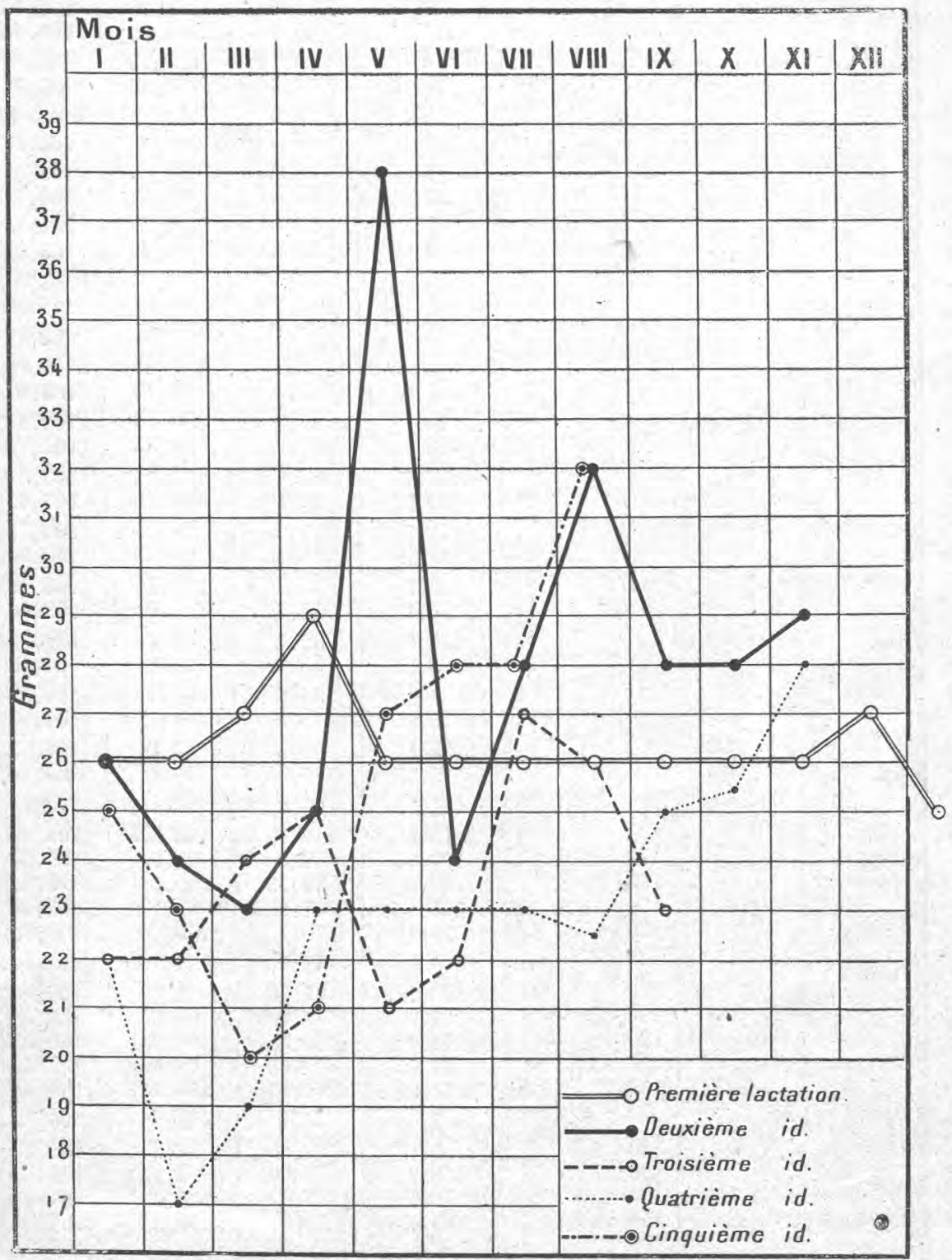

Tiıx butyreux moyen mensuel du lait de la même chèvre pendant cinq lactations consécutives (Institut Zoolechnique de l'Ecole vétérinaire de Dresde). - Si ces cinq courbes sont assez ramassées, elles n'en ont pas moins chacune leur allure propre différente de celles des_autres. 
25 arril Mi

\begin{tabular}{|c|c|c|c|}
\hline $\begin{array}{l}\text { EXTRAIT } \\
\text { sec total }\end{array}$ & $\begin{array}{c}\text { MATIÈRE } \\
\text { grasse }\end{array}$ & $\begin{array}{l}\text { EXTRAIT } \\
\text { dégraissé } \\
\text { brut }\end{array}$ & $\begin{array}{c}\text { EXTRAIT } \\
\text { dégraissé } \\
\text { rectifié }\end{array}$ \\
\hline $\begin{array}{l}142,25 \\
145,30\end{array}$ & $\begin{array}{l}40,65 \\
44,5\end{array}$ & $\begin{array}{l}101,6 \\
100,8\end{array}$ & $\begin{array}{l}104,75 \\
105,00\end{array}$ \\
\hline $\begin{array}{l}130,2 \\
151,1\end{array}$ & $\begin{array}{l}30,45 \\
52,4\end{array}$ & $\begin{array}{l}99,75 \\
99,30\end{array}$ & $\begin{array}{l}103,00 \\
105,15\end{array}$ \\
\hline $\begin{array}{l}130,2 \\
147,25\end{array}$ & $\begin{array}{l}31,5 \\
46,9\end{array}$ & $\begin{array}{r}99,15 \\
100,35\end{array}$ & $\begin{array}{l}102,5 \\
105,60\end{array}$ \\
\hline $\begin{array}{l}139,9 \\
138,95\end{array}$ & $\begin{array}{l}38,15 \\
38,6\end{array}$ & $\begin{array}{l}100,75 \\
100,35\end{array}$ & $\begin{array}{l}105,0 \\
104,8\end{array}$ \\
\hline $\begin{array}{l}138,6 \\
149,9\end{array}$ & $\begin{array}{l}38,0 \\
49,0\end{array}$ & $\begin{array}{l}100,6 \\
100,0\end{array}$ & $\begin{array}{l}104,9 \\
105,7\end{array}$ \\
\hline $\begin{array}{l}135,1 \\
153,0\end{array}$ & $\begin{array}{l}36,05 \\
54,35\end{array}$ & $\begin{array}{l}99,05 \\
98,65\end{array}$ & $\begin{array}{l}103,0 \\
104,8\end{array}$ \\
\hline $\begin{array}{l}137,4 \\
150,3\end{array}$ & $\begin{array}{l}37,00 \\
50,85\end{array}$ & $\begin{array}{r}100,4 \\
99,45\end{array}$ & $\begin{array}{l}104,5 \\
105,2\end{array}$ \\
\hline $\begin{array}{l}136,6 \\
144,3\end{array}$ & $\begin{array}{l}37,4 \\
43,75\end{array}$ & $\begin{array}{r}99,25 \\
100,55\end{array}$ & $\begin{array}{l}103,5 \\
105,40\end{array}$ \\
\hline 128,2 & 27,4 & 100,8 & 103,8 \\
\hline $\begin{array}{l}132,85 \\
141,6\end{array}$ & $\begin{array}{l}32,45 \\
43,3\end{array}$ & $\begin{array}{c}100,4 \\
98,30\end{array}$ & $\begin{array}{l}104,0 \\
103,8\end{array}$ \\
\hline $\begin{array}{l}117,6 \\
118,95\end{array}$ & $\begin{array}{l}17,5 \\
19,30\end{array}$ & $\begin{array}{r}100,55 \\
99,65\end{array}$ & $\begin{array}{r}102,4 \\
101,6\end{array}$ \\
\hline $\begin{array}{l}126,7 \\
131,25\end{array}$ & $\begin{array}{l}26,45 \\
31,1\end{array}$ & $\begin{array}{l}100,25 \\
100,15\end{array}$ & $\begin{array}{l}103,25 \\
103,65\end{array}$ \\
\hline $\begin{array}{l}113,7 \\
116,95\end{array}$ & $\begin{array}{l}14,45 \\
17,90\end{array}$ & $\begin{array}{l}99,25 \\
99,05\end{array}$ & $\begin{array}{l}100,75 \\
100,90\end{array}$ \\
\hline $\begin{array}{l}122,6 \\
125,9\end{array}$ & $\begin{array}{l}21,75 \\
25,30\end{array}$ & $\begin{array}{r}100,85 \\
100,60\end{array}$ & $\begin{array}{l}103,25 \\
103,40\end{array}$ \\
\hline $\begin{array}{l}131,8 \\
145,25\end{array}$ & $\begin{array}{l}32,7 \\
45,65\end{array}$ & $\begin{array}{l}99,10 \\
99,6\end{array}$ & $\begin{array}{l}102,75 \\
104,75\end{array}$ \\
\hline $\begin{array}{l}137,4 \\
145,25\end{array}$ & $\begin{array}{l}36,65 \\
45,80\end{array}$ & $\begin{array}{r}100,75 \\
99,45\end{array}$ & $\begin{array}{l}104,85 \\
104,60\end{array}$ \\
\hline $\begin{array}{l}154,8 \\
175,25\end{array}$ & $\begin{array}{l}56,8 \\
75,25\end{array}$ & $\begin{array}{l}98,00 \\
96,00\end{array}$ & $\begin{array}{l}104,45 \\
104,35\end{array}$ \\
\hline 162,55 & 64,2 & 98,45 & 105,8 \\
\hline 213,6 & 119,75 & 93,85 & 108,20 \\
\hline 178,75 & 80,8 & 97,95 & 107,00 \\
\hline 182,8 & 83,75 & 99,05 & 108,85 \\
\hline 165,8 & 65,9 & 99,9 & 107,40 \\
\hline 170,1 & 70,1 & 100,00 & 108,00 \\
\hline 148,55 & 47,45 & 101,10 & 106,40 \\
\hline 193,2 & 96,20 & 97,0 & 106,70 \\
\hline 178,95 & 79,9 & 99,05 & 108,00 \\
\hline 164,2 & 65,0 & 99,2 & 106,65 \\
\hline 158,65 & 59,05 & 99,60 & 106,50 \\
\hline 167,20 & 66,05 & 101,15 & 108,80 \\
\hline 136,6 & 34,4 & 102,2 & 106,30 \\
\hline 188,1 & 91,85 & 96,25 & 106,25 \\
\hline 171,25 & 73,10 & 98,15 & 106,5 \\
\hline 153,6 & 53,6 & 100,0 & 106,2 \\
\hline 155,35 & 56,0 & 99,35 & 105,9 \\
\hline 146,5 & 46,8 & 99,7 & 105,2 \\
\hline $\begin{array}{l}137,9 \\
156,55\end{array}$ & $\begin{array}{l}38,0 \\
60,85\end{array}$ & $\begin{array}{l}99,9 \\
96,0\end{array}$ & $\begin{array}{l}104,4 \\
102,70\end{array}$ \\
\hline 139,4 & 42,5 & 96,9 & 101,9 \\
\hline 150,2 & 53,2 & 97,0 & 102,9 \\
\hline 144,3 & 46,85 & 97,45 & 102,6 \\
\hline 164,2 & 67,25 & 96,95 & 104,45 \\
\hline 138,25 & 40,8 & 97,45 & 101,9 \\
\hline 138,30 & 40,35 & 97,95 & 102,35 \\
\hline
\end{tabular}


Je joins, en outre, à ce court article qui ne renferme que quelques aperçus sur le lait de chèvre, des courbes qui ont été dispersées dans le texte pour la commodité de la disposition typographique et dont l'examen, mieux que tout développement écrit, montrera l'intérêt.

Je terminerai en donnant un tableau de composition moyenne du lait de chèvre et de celle du lait de vache :

\begin{tabular}{|c|c|c|}
\hline & $\begin{array}{l}\text { Vache } 0 / \% 0 \\
\text { (en poids) }\end{array}$ & $\begin{array}{l}\text { Chèvre } \% \\
\text { (en poids) }\end{array}$ \\
\hline & - & - \\
\hline$\ldots \ldots \ldots \ldots \ldots \ldots \ldots \ldots \ldots$ & 875 & 870 \\
\hline Extrait sec $\ldots \ldots \ldots \ldots \ldots \ldots$ & 125 & 130 \\
\hline Matière grasse $\ldots \ldots \ldots \ldots \ldots \ldots \ldots$ & 37 & 40 \\
\hline Lactose....................... & 48 & 48 \\
\hline Matières | caséine .......... & $28-30$ & $31-32$ \\
\hline protéiques a albumine........... & $4-5$ & $7-8$ \\
\hline Cendres........................ & 7 & 7 \\
\hline
\end{tabular}

\section{BIBLIOGRAPHIE ANALYTIQUE}

\section{$1^{\circ}$ LIVRES.}

Joseph Crepin. - La chèvre, son histoire, son élevage pratique, ses bienfaits, ses services. Préface de M. Edmond Perrier, 2 édit., 1 vol. broché de 333 pages, avec de nombreuses photographies. Librairie Hachette et Cie, Paris, $10 \mathrm{fr}$.

Le livre de C. est le iseul que nous possédions actuellement dans la littérature française sur la chèvre. C'est, comme le dit le préfacier, une œuvre d'apostolat à laquelle on ne saurait trop applaudir.

La chèvre ñ'est pas ce que l'on croit générälement, et C. s'emploie à la montrer sous son véritable jour, dégagée de tous les préjugés qui affaiblissent l'idée que l'on se fait communément de sa nature et 'de ses qualités, Il est vraisemblable que la chèvre a été, et est encore aujourd'hui, la première bête laitière que l'homme ait connue et utilisée. Elle alimente presque exclusivement de son lait beaucoup de populations du Sud de l'Europe et celles de tout le continent africain.

Ce qui a jeté principalement le discrédit sur la chèvre, c'est son goût prononcé pour les végétaux ligneux. Les dégâts qu'elle cause aux arbres fruitiers, ses déprédations dans les forèts, l'ont vouée au ressentiment des agriculteurs et des agents des forêts.

Une autre cause de discrédit est ce " perpétuel et énervant » qualificatif 